\title{
MGMT-B Gene Promoter Hypermethylation in Patients with Inflammatory Bowel Disease - A Novel Finding
}

\author{
Pooneh Mokarram ${ }^{1}$, Soudabeh Kavousipour ${ }^{1}$, Mostafa Moradi Sarabi ${ }^{1}$, Golnosh \\ Mehrabani' $^{1}$, Mohammad Ali Fahmidehkar', Seyedeh Azra Shamsdin ${ }^{1}$, Abbas \\ Alipour ${ }^{2}$, Mahvash Alizade Naini ${ }^{3 *}$
}

\begin{abstract}
Inflammatory bowel disease (IBD) is a disease strongly associated with colorectal cancer (CRC) as a wellknown precancerous condition. Alterations in DNA methylation and mutation in K-ras are believed to play an early etiopathogenic role in CRC and may also an initiating event through deregulation of molecular signaling. Epigenetic silencing of APC and SFRP2 in the WNT signaling pathway may also be involved in IBD-CRC. The role of aberrant DNA methylation in precancerous state of colorectal cancer (CRC) is under intensive investigation worldwide. The aim of this study was to investigate the status of promoter methylation of MGMTB, APC1A and SFRP2 genes, in inflamed and normal colon tissues of patients with IBD compared with control normal tissues. A total of 52 IBD tissues as well as corresponding normal tissues and 30 samples from healthy participants were obtained. We determined promoter methylation status of MGMT-B,SFRP2 and APC1A genes by chemical treatment with sodium bisulfite and subsequent MSP. The most frequently methylated locus was MGMT-B (71\%; 34 of 48), followed by SFRP2 (66.6 \%; 32 of 48), and APC1A (43.7\%; 21 of 48). Our study demonstrated for the first time that hypermethylation of the MGMT-B and the SFRP2 gene promoter regions might be involved in IBD development. Methylation of MGMT-B and SFRP2 in IBD patients may provide a method for early detection of IBD-associated neoplasia.
\end{abstract}

Keywords: Inflammatory bowel disease - colorectal cancer - gene methylation

Asian Pac J Cancer Prev, 16 (5), 1945-1952

\section{Introduction}

Inflammatory bowel disease (IBD) with at least two subtype diseases; ulcerative colitis (UC) and Crohn's disease $(\mathrm{CD})$ is strongly associated with uncontrolled chronic inflammation of intestines (Hartnett and Egan, 2012). Its incidence is 30/000 worldwide and has both acute and chronic mild to severe complication with high burden on quality of life (QoL) and economic status (Hanauer, 2006). Chronic Inflammation is thought to be the root cause of tumor development and associated with about $20 \%$ of all human cancers (Ullman and Itzkowitz, 2011; Hartnett and Egan, 2012). The most serious associated chronic complication of IBD is colorectal cancer (CRC); IBD patients are $60 \%$ more likely prone to develop CRC than general population with $10-15 \%$ mortality (Munkholm, 2003; Goel et al., 2011; Herrinton et al., 2012). So cancer surveillance is mandatory in these patients.
Screening of IBD patients by repeated colonoscopy and intestinal biopsy as an invasive and expensive approach is time consuming, inefficient and not feasible for all IBD patients. In order to overcome such difficulties, intensive investigation are done by researchers to find noninvasive and good assessable diagnostic tools to be well-validated for early detection, prognosis and monitoring of precancerous state (Chambers et al., 2005; Mattar et al., 2011).

Epigenetic alterations, particularly alteration in DNA methylation are believed to play an early etiopathogenic role in IBD (Petronis and Petroniene, 2000; Tahara et al., 2009; Lin et al., 2011; Cooke et al., 2012; Hartnett and Egan, 2012; Olaru et al., 2012).

DNA hypermethylation is associated with gene silencing and is often observed in $\mathrm{CpG}$ islands of cancer-relatedted genes in CRC and other cancers (Fang et al., 2012; Wani et al., 2012; Al-Shabanah et al., 2014; Sangplod et al., 2014). Aberrant cancer specific

${ }^{1}$ Department of Biochemistry, Gastroenterohepatology Research Center, Nemazee Hospital, Colorectal Research Center, ${ }^{3}$ Department of Internal Medicine, School of Medicine, Shiraz University of Medical Sciences, Shiraz, ${ }^{2}$ Social Medicine Department, Medical Faculty, Mazandaran University of Medical Sciences, Sari, Iran*For correspondence: alizadem@sums.ac.ir, malizadenaini@ yahoo.com 
methylation of genes like p16, E-cadherin, hMLH1 and p14 has been reported in IBD associated neoplasia (Dhir et al., 2008; Low et al., 2013). These epigenetic changes may accelerate the development of IBD-CRC (Petronis and Petroniene, 2000; Goel, et al., 2011; Jawad et al., 2011; Lin, et al., 2011; Jenke and Zilbauer, 2012; Kellermayer, 2012). CpG island methylation phenotype and global DNA methylation with LINE-1 assay were also observed in $17 \%$ and $58 \%$ of the ulcerative colitis (UC) related cancers, respectively (Konishi et al., 2007). Loss of hMLH1 protein expression associated with gene hypermethylation is common in Microsatellite instability (MSI) positive UC cancers (Fleisher et al., 2000). Methylation of hyperplastic polyposis gene 1 (HPP1) was observed in $50 \%$ UC adenocarcinoma and $40 \%$ of dysplasias but not in normal mucosa (Sato et al., 2002). Also, methylation of E-cadherin (CDH1) was detected in $93 \%$ of the patients with dysplasias (Azarschab et al., 2002). In addition, researchers reported that the increased level of methylation are widespread in the inflammation affected colon and occur early in the process of carcinogenesis (Issa et al., 2001).

On the other hand the role of Wnt signaling pathway at different stages of gut development, as well as during adult epithelial homeostasis is known and the association of mutational deregulation with malignant transformation is documented also. Aberrant WNT signalling pathway as an early progression event has crucial roles for cancer progression (Anastas and Moon, 2013). Epigenetic alteration of WNT signaling genes has been reported in up to $80 \%$ of patients with CRC (Barker and Clevers, 2006; Dhir, et al., 2008; You et al., 2008; Lin, et al., 2011). Interestingly, differential methylations in tissues of IBD patients were identified, suggesting that IBD-associated changes in DNA methylation may be disease subtype specific. Wnts Ligands initiate the Wnt signaling cascade by interacting with the Fz receptor and LRP co-receptor (Anastas and Moon, 2013). These interactions subsequently lead to formation of the complex composed of APC, AXIN and Glycogen synthase kinase$3 \beta$ (GSK-3 $\beta$ ). Together, APC/AXIN/GSK-3 $\beta$ make up the $\beta$-catenin destruction complex. In the absence of Wnts stimulation, the APC/AXIN/GSK- $3 \beta$ complex phoshphorylate $\beta$-catenin. Phosphorylated $\beta$-catenin then recognized by $\beta$-TrCP protein and target for proteosomal degradation (Baylin and Ohm, 2006). $\beta$-catenin is the cytoplasmic protein and the key component of the Wnt signaling pathway. In the presence of wnts stimulation, or loss of APC due to mutation, the destruction complex is destabilized, resulting in excessive amounts of $\beta$-catenin. $\beta$-catenin then translocates to the nucleus, where it associates with the Tcf family of transcription factors and promotes the transcription of many target genes including c-MYC and cyclin D1 whose products increase cell proliferation (Noah et al., 2013). In addition, there is crosstalk between STAT3 with WNT signaling pathway during pathogenesis of CRC (Pandurangan and Esa, 2014). Secreted Frizzled-related proteins (SFRPs) are tumor suppressor genes that function as antagonists of WNT signaling. SFRP2 bind to Wnts and preventing their interaction with $\mathrm{Fz}$ and LRP (Baylin and Ohm, 2006). Dysregulation of WNT signaling pathway owing to epigenetic silencing of key tumor suppressor genes such as APC and SFRP2 may be involved in IBD-CRC (Dhir, et al., 2008). Rarity of WNT signaling genes mutation and limited studies addressing the effects of WNT genes methylation in IBD associated colorectal cancer mandate more research in this field.

The other known mechanism for carcinogenesis is activation of the Raf/MEK/ERK (MAPK) kinase pathway through either Kras or BRAF mutation which was detected in nearly $30 \%$ of UC related cancers. Non-dysplastic mucosa of patients with UC induced colorectal cancers shows Kras but not BRAF mutation, indicating that Kras mutations are an initiating event in UC carcinogenesis (Aust et al., 2005). Several studies were also reported that reduced expression and function of DNA repair enzyme induced by oxidative stress during inflammation disease (Switzeny et al., 2012). DNA Mismatch repair elements and O6-methylguanine-DNA methyltransferase (O6-MGMT) are DNA repair proteins. MGMT loss of activity associated with creating a permissive state for $\mathrm{G}>\mathrm{A}$ mutations in protooncogenes such as Kras and p53 (Mokarram et al., 2013). Kras mutations occur in about $60 \%$ of CRCs and are an early event in UC carcinogenesis (Aust, et al., 2005; Lao and Grady, 2011).

New but limited insight about loss of activity of DNA repair proteins and the state of MGMT methylation mandate more research both in CRC and in precancerous states such as IBD and polyps.

MGMT promoter has two loci (Lin, et al., 2011; Mokarram, et al., 2013) which we described as MGMT-A

Table 1. Primers Sequence of SFRP2, MGMT and APC1A

\begin{tabular}{lllcc}
\hline Gene & Gene Bank No. & Primer sequence (5 a 3) & Annealing Temperature $\left({ }^{\circ} \mathrm{C}\right)$ & Product size (bp) \\
\hline SFRP2 & NM_003013.2 & MF: TGCGTGTTTTATTTCGTAGTTCGC & 59 & M: 138 \\
& & MR: CCCTAAATACCGCCGCTCGCCCG & U: 145 \\
& & UF: GTTTTGTGTGTTTTATTTTGTAGTTTG & & M: 97 \\
APC1A & NM_022662.3 & MF: TATTGCGGAGTGCGGGT & & U: 112 \\
& & MR: TCGACGAACTCCCGACG & 63 & M: 127 \\
& & UF: GTGTTTATTGTGGAGTGTGGGT & & U: 127 \\
MGMT-B & UR: CCAATCAACAAACTCCCAACA 355531.16 & MF: GGTCGTTTGTACGTTCGC & & \\
& & MR: TAACCCTTCGACCGATACAA & 59 & \\
& & UF: GTAGGTTGTTTGTATGTTGT & & \\
\hline
\end{tabular}


and MGMT-B in our recent research (Mokarram, et al., 2013). Since, methylation silencing of MGMT-B rather than MGMT-A was significantly associated with Kras gene mutation in CRC patients but not in normal tissues (Mokarram, et al., 2013), we hypothesized that methylation of MGMT-B as caretaker gene may be responsible for dysregulation of signaling through Kras mutation in IBD patients.

Therefore, epigenetic alterations of APC1A, SFRP2 and MGMT-B genes may be seen in IBD patients with a probable potential to be used as a reliable clinical biomarkers for diagnostic, prognostic and therapeutic applications of IBD-CRCs.

\section{Materials and Methods}

\section{Patients and tumor specimens}

Of total 82 IBD patients undergoing colonoscopic evaluation at clinical centers affiliated to Shiraz University of Medical Sciences, Southern Iran, during 2 years (20112013), 78 had recent onset disease and 52 had criteria to be included in this research; new onset was not previously treated and histological evaluation by an expert pathologist documented clinico-pathological of IBD patients. 52 IBD samples as well as corresponding normal tissues were obtained from patients. Also 30 age and sex matched healthy participants were selected from 80 volunteers who performed colonoscopy and had normal colonic mucosa. Individuals with eligible criteria who provided written informed consent were enrolled. Ethics committee and institutional review board of Shiraz University of Medical Sciences approved the study. All Fresh samples were snapfrozen and stored at $-70^{\circ} \mathrm{C}$ until processing.

Extraction of DNA

Genomic DNA was extracted from samples, as described previously (Mokarram et al., 2008).We used the standard phenol/chloroform method for DNA extraction from fresh tumor samples (Mokarram, et al., 2008).

\section{Methylation specific PCR}

We determined promoter methylation status of MGMT, SFRP2 and APC1A genes by chemical treatment with sodium bisulfite and subsequent MSP, as described (Mokarram, et al., 2008). In brief, this technique uses bisulfate modification to convert unmethylated, but not methylatedcytosine to uracil. MSP utilizes this difference to amplify specifically either methylated or unmethylated DNA.

Locus specific PCR primers for three genes MGMT-B, SFRP2,APC1A were specifically designed for methylation specific PCR and located at each gene promoter region. The sequences, annealing temperature of each primer used for amplification and PCR products size were described in Table 1.

The hot-start PCR reactions were performed in a $50 \mu \mathrm{L}$ reaction volume containing 25 pmol of sense and antisense primers, $0.2 \mathrm{mmol} / \mathrm{L} \mathrm{dNTPs}$ and $80 \mathrm{ng}$ bisulfite-modified DNA in 1 X PCR buffer provided by Taq enzyme supplier. The reaction mixture was denatured at $95^{\circ} \mathrm{C}$ for $5 \mathrm{~min}$, after which $1.5 \mathrm{U}$ Taq polymerase was added; then amplified by 40 cycles, each consisting of $30 \mathrm{~s}$ denaturation at $95^{\circ} \mathrm{C}$, proper annealing temperature for each gene [Table 1] and $30 \mathrm{~s}$ polymerization at $72^{\circ} \mathrm{C}$, followed by a single 10 -min extension at $72^{\circ} \mathrm{C}$. The universal methylated DNA (chemicon) was used as positive control for methylated alleles of MGMT-B, SFRP2 and APC1A and DNA from normal lymphocytes was used as the negative control. $10 \mu \mathrm{L}$ of amplified PCR products were mixed with $5 \mu \mathrm{L}$ of loading dye and electrophoresed on $2.5 \%$ agarose gel containing gel red with TBE buffer and visualized under UV illumination.

\section{Statistical analysis}

Depending upon the sample size, associations between clinical, biological and genotypic features were evaluated using either the Chi square test or the Fisher's exact test. The level of significance was assessed at $p<0.05$. We also studied potential confounding variables such as age and gender. All statistical data were analyzed by SPSS software, version 11.5 (SPSS Inc., Chicago, IL).

\section{Results}

Distribution of selected IBD patients and controls

According to clinical symptom, 52 patients were included from two referral hospitals in Shiraz, south of Iran in this study. Colonoscopy and biopsy sampling from IBD lesions and corresponding adjacent normal was performed in all patients. 4 out of 52 were excluded from the study due to their pathological results that no significant changes were reported. 30 individuals that were referred to colonoscopy but no IBD changes were reported in their results were selected as normal controls.

\section{Prevalence of MGMT-B, APC1 $a$ and SFRP2:}

48 patients were analyzed for methylation of MGMT-B, SFRP2 and APC1A in this study. Distributions of selected clinical-biological characteristics of total patients are presented in table 2. The frequencies of the methylation status of the three promoters and their relationship with the clinical characteristics of the patients were summarized in Table 3.

Several studies have reported age-dependent methylation (Mokarram, et al., 2008), therefore, we divided both IBD and control groups into $\geq 29$ and $<29$ year old groups. The median age of IBD patients (29years) was chosen for this division. Our data showed that neither gender, nor age was associated with the promoter methylation status of the genes.

No methylation of MGMT-B, APC1A and SFRP2

Table 2. Demographic Data of Samples

\begin{tabular}{|c|c|}
\hline Variables & Total \\
\hline \multicolumn{2}{|l|}{ Gender } \\
\hline Male & $18(37.5 \%)$ \\
\hline Female & $30(62.5 \%)$ \\
\hline Total & $48(100 \%)$ \\
\hline \multicolumn{2}{|l|}{ Age groups } \\
\hline$<29$ & $25(52.1 \%)$ \\
\hline${ }^{3} 29$ & $23(47.9 \%)$ \\
\hline Median, range & $29(11-55)$ \\
\hline Mean ( \pm SD) & $31.6 \pm 10.5$ \\
\hline
\end{tabular}


Table 3. Results fo Methylation Status

\begin{tabular}{|c|c|c|c|c|c|c|c|c|c|}
\hline \multirow[t]{2}{*}{ Variables } & \multicolumn{6}{|c|}{ Methylation Positive } & \multicolumn{2}{|c|}{ At least one Methylation } & At least one methylation \\
\hline & APC1A & $\mathrm{p}$ & SFRP2 & $\mathrm{p}$ & MGMT & $\mathrm{p}$ & $\begin{array}{l}\text { SFRP2/ } \\
\text { APC1A }\end{array}$ & $\mathrm{p}$ & $\begin{array}{l}\text { SFRP2/ p } \\
\text { APC1A/ } \\
\text { MGMT }\end{array}$ \\
\hline \multicolumn{10}{|l|}{ Sex } \\
\hline Male & $7(41.2 \%)$ & & $1(68.8 \%)$ & & $12(66.6)$ & & $13(86.7 \%)$ & & $16(88.9)$ \\
\hline Female & $13(43.3 \%)$ & 1 & $21(70 \%)$ & 1 & $21(73.3)$ & 0.3 & $25(83.3 \%)$ & 1 & $28(93.3)^{0.6}$ \\
\hline \multicolumn{9}{|l|}{ Age } & 44(91.7) \\
\hline$<29$ & $9(37.5 \%)$ & & $16(66.7 \%)$ & & $18(75)$ & & $19(82.6 \%)$ & & $23(92)$ \\
\hline 329 & $11(47.8 \%)$ & 0.5 & $16(72.7 \%)$ & 0.7 & 16(66.6) & 0.5 & 19(86.4\%) & 1 & $21(91.3)^{1}$ \\
\hline
\end{tabular}

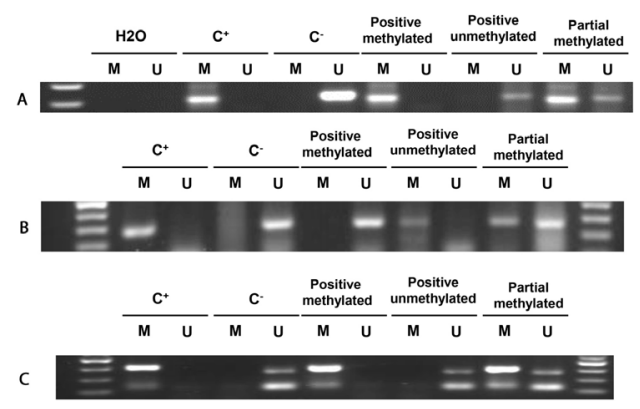

Figure 1. Representative examples of MSP reactions for promoter methylation analysis of $\operatorname{APC1A}(\mathrm{A})$, MGMT-B (B) and SFRP2 (C) genes in primary CRC tumors. The presence of a visible PCR product in those lanes marked $U$ indicates the presence of unmethylated genes; the presence of a product in those lanes marked $M$ indicates the presence of methylated genes. Lane 1 indicates the $50 \mathrm{bp}$ DNA size marker. Universal methylated DNA (UMD) and unmethylated lymphocytes (lymphocytes) DNA was used as positive and negative controls.

were found in normal tissues of 30 healthy people and corresponding adjacent normal mucosa from our patients.

The most frequently methylated locus was MGMT (71\%; 34 of 48), followed by SFRP2 (66.6\%; 32 of 48), and APC1A (43.7\%; 21 of 48). The frequency of promoter methylation in IBD patients with at least one of 3 genes methylated was 44/48 (91.7\%).

41 of $48(85.4 \%)$ of IBD patients had MGMT-B or SFRP2 hypermethylation and 38 of $48(84.4 \%)$ patients had methylation of APC1A or SFRP2 while 38 of $48(79.2 \%)$ had MGMT-B or APC1A promoter hypermethylation.

The frequency of methylation for both SFRP2 and APC1A, MGMT and SFRP2, MGMT and APC1A methylation were 14/48 (29.2), 21/48 (43.8), 13/48 (27.1), respectively. Ten of the IBD samples had simultaneous $\mathrm{CpG}$ island hypermethylation of all three genes. There were no significant differences in association of methylation of any individual gene investigated by age or sex of patients. Some examples of MGMT-B, SFRP2 and APC1A methylation are shown in Fig. 1.

\section{Discussion}

Our study demonstrates for the first time hypermethylation of the MGMT-B gene and the SFRP2 gene promoter region as antagonist of WNT signaling were involved in IBD developing lesions. However, we have not shown a significant increase in methylation of APC1A gene from the WNT signaling pathway genes during the development of IBD lesions.

Since epigenetic changes can also be detected in precancerous lesions tissues (Gonzalo et al., 2010) suggesting its potential role in the initial carcinogenesis (Petronis and Petroniene, 2000; Jawad, et al., 2011; Cooke, et al., 2012; Jenke and Zilbauer, 2012; Olaru, et al., 2012). Gene promoter hypermethylation in normal appearing colorectal mucosa has been suggested in the MGMT gene as well as, MLH1, TIMP3 and DAPK (Gonzalo, et al., 2010).

The prevalence of gene silencing mediated by hypermethylation in IBD remains controversial, however considering the variable gene methylation prevalence reported in IBD-CRCs (Fleisher, et al., 2000; Konishi, et al., 2007; Dhir, et al., 2008; Cooke, et al., 2012; Olaru, et al., 2012).

Recent studies showed that there is a link between IBD-associated inflammation and carcinogenesis when increased abnormal $\mathrm{CpG}$ island hypermethylationmediated gene silencing hasbeen occurred (Azarschab, et al., 2002; Sato, et al., 2002; Dhir, et al., 2008).

Evidence shows that anti-oxidants are able to increase DNA repair enzyme activities (Waris and Ahsan, 2006; Hegde et al., 2008; Switzeny, et al., 2012). Reactive oxygen species which is produced during each inflammation such as Inflammatory bowel disease induced spontaneous deamination of cytosine to uracil and 5-hydroxyuracil leads to a G:C to T:A transition mutation, due to its mispairing with adenosine (Switzeny, et al., 2012). Therefore, DNA integrity and DNA repair enzymes are crucial especially in IBD patients. MGMT removes alkyl adducts from the DNA especially O6methylguanine, which is read as an adenine by the DNA polymerase (Mokarram, et al., 2013). Our promising results in recent publication showed that MGMT-B methylation is significantly associated with Kras gene mutation rather than MGMT-A (Mokarram, et al., 2013). Defect in MGMT leads to $G$ to A transition mutation which shown in CRC patients with K-ras mutation (Mokarram, et al., 2013). Methylation of O-6-methylguanine DNA methyltransferase (MGMT), is also linked with low-level MSI- (MSI-L) colorectal cancer (Whitehall et al., 2001; 
Psofaki et al.,2010). MGMT-A promoter is inactivated by hypermethylation, and a promoter methylation-dependent downregulationof the corresponding gene expression in some cancer tissues has been found (Jain and Peppercorn, 1997; Piperi et al., 2010).

There is little information about methylation of each CpG of MGMT gene in IBD patients, only 2 studies showed that low frequency of MGMT methylation in ulcerative colitis associated tumors occurred than sporadic colonic tumors (Matsumura et al., 2003; Mikami et al., 2007). According to high frequency of MGMT-B methylation in our CRC patients and increasing rate of CRC in our country, one of our primary aims was to assess the epigenetic pattern of MGMT-B in IBD patients.

The discrepancy may be due to the location of methylation as we showed in case of MGM density of $\mathrm{CpG}$ sites is not critical for CRC development and MGMT-B that was located in the body of the gene methylated more than MGMT-A patients with CRC (Mokarram, et al., 2013).

In this study, we show that hypermethylation of MGMT-B has also the ability to predispose patients to IBD lesions. The higher rates of MGMT-B methylation compared to SFRP2 and APC1A showed the methylation of MGMT-B is more crucial than WNT signaling pathway in development of IBD.

Although we employed the MSP assay to detect methylated MGMT DNA in the biopsy samples of IBD patients, we were able to detect methylated DNA in serum samples only for the MGMT promoter in all patients(data not shown) suggesting that DNA methylation of MGMT might be a pivotal event in the development IBD lesions to CRC. Furthermore, the aberrant methylation of MGMT has been shown to silence some genes and to result in cancer promoting events, such as an MSI-L phenotype or Kras mutation (Svrcek et al., 2010; Mokarram, et al., 2013). Also, recent study showed that MGMT defects occurs more in MSI than MSS CRC phenotypes. These facts indicate that methylation of MGMT is superior initiating step to MMR deficiency in MSI CRC phenotype development in sporadic and IBD patients (Svrcek, et al., 2010).

On the other hand, the involvement of WNT signaling pathway in pathogenesis of CRC has been proved. Although mutation in APC is seen in more than $80 \%$ of CRC patients, but mutation of WNT signaling genes in IBD patients are rare (Dhir, et al., 2008). Therefore, it seems that the role of epigenetic alteration of WNT signaling pathway cannot be ruled out. In this study, we evaluated the methylation of SFRP2 and APC1A as the most valuable and important genes in WNT signaling pathway. Higher rate of methylation in SFRP2 compared with APC1A show that the methylation of SFRP2 is the second early marker after MGMT seen in our patients with IBD.

38 of cases $(84.4 \%)$ had methylation in at least one of these genes and 12 patients $(25 \%)$ had methylation in both of the genes. According to data, this study showed strong relation between IBD and these two genes, especially SFRP2. We have shown that methylation of SFRP2 or APC1A might be first starts in IBD patients in our region.
Therefore, we suggest a strong role of methylation of two WNT signaling pathway genes in the pathogenesis of IBD.

In this regards, Herman et al showed high levels of methylation of the WNT signaling pathway genes in early lesions like low-grade dysplasias (LGDs) in IBD patients (Dhir, et al., 2008). It seems that methylation is seen overall in inflamed mucosa higher than non-inflamed normal colon. Therefore, inflammation can start chronic injury through tumor related genes by methylation as individuals with cirrhosis predispose to hepatocellular cancer (Okochi et al., 2003). Since methylation of APC1A is more common than mutation, epigenetic inactivation may be a predominant mechanism for inactivation of APC1A and subsequent development of cancer in IBD patients (Dhir, et al., 2008). Our finding was inconsistent when we compared the frequency of APC1A in our patients with the only available study. Biologically different in colorectal cancer which depends on life style variables and geographic differences could possibly answer why frequency of APC1A methylation differ in our population compared with Herman's study. Studies showed that p53 mutations occur early and are more frequent in non-dysplastic mucosa in ulcerative colitis associated neoplasia (Burmer et al., 1992; Yin et al., 1993; Brentnall et al., 1994). Interestingly, APC2 were associated in interaction with the $\mathrm{P} 53 / \mathrm{Bcl} 2$ in cell cycle progression and cell death pathway (Nakagawa et al., 2000). It is very interesting to be viewed the APC2and P53 interaction pathway in the IBD patients. We performed APC2 methylation and higher methylation than APC1a observed in our study (data not shown).

On the other hand, there is a strong link between MGMT methylation and p53 and Kras mutation (Wu et al., 2008; Shamsara et al., 2009; Mokarram, et al., 2013).

Researchers showed that early dysplastic lesions show a high level of p53 mutation and $\mathrm{Bcl} 2$ over expression (Yin, et al., 1993; Brentnall, et al., 1994; Bronner et al., 1995). Interestingly, we have found MGMT-B to be methylated to high levels in IBD lesions. Therefore, MGMT-B methylation might play a promising role in the pathogenesis of these lesions.

On the other hand SFRPs can inhibit the WNT signaling pathway either by interacting with WNT proteins or by forming complexes with frizzled receptors (Dhir, et al., 2008). Therefore, the frequent inactivation of SFRP2 through methylation can highlight activation of WNT signaling pathway in the pathogenesis of our IBD patients.

Since dysplasia and inflammation are as precursor for existing malignancy in IBD patients (Jain and Peppercorn, 1997; Zisman and Rubin, 2008; Mattar, et al., 2011; Zisman et al., 2012; Harpaz et al., 2013). So diagnosis of these lesions by molecular analysis is prime goals that reduce mortality rate from CRC associated IBD and provide a sensitive markers in clinical management of patients with IBD. Our study demonstrates for the first time epigenetic involvement of DNA repair gene (MGMT) and the two WNT signaling pathway genes in our IBD patients. According to our findings, we can use these two genes (MGMT-B, SFRP2) to early detection and prevention of colorectal cancer in IBD cases.

Recently, researchers notice to cell free methylated 
DNA based biomarkers in serum or stool (Widschwendter and Menon, 2006; Bosch et al., 2012; Philipp et al., 2012; Zhai et al., 2012; Hong and Ahuja, 2013; Summers et al., 2013). DNA shed into serum or stool provides a more accurate sampling of abnormal cells than random punch biopsies among IBD associated cancer patients.

Stool DNA testing for hypermethylation of the SFRP-1 promoter has already been shown to be a sensitive and specific screening tool for sporadic CRC (Zhang et al., 2007). Several studies also suggest that methylated DNA released in the circulation could be used as a prognostic marker for early tumor detection. The presence of aberrantly methylated genes such as SEPT9, HLTF and HPP1 DNA in plasma is highly correlated with the occurrence of colorectal cancer and tumor size, stage, grade and metastatic disease, respectively (deVos et al., 2009; Herbst et al., 2009; Lange et al., 2012; Summers, et al., 2013).

Our findings of methylation of MGMT-B and SFRP2, if validated in future studies, could be used as serum or stool-based DNA methylation tools for early detection of IBD patients before existing cancer. Moreover, early methylation of MGMT-B, SFRP2, APC1A in IBD patients may provide a method for early detection of IBD-associated neoplasia which will be independent of the pathologist. Further studies will be required to validate the findings of the current study as valuable and promising markers.

\section{Acknowledgements}

This study was supported by grant number 4760 from Vice-Chancellor for Research Affairs of Shiraz University of Medical Sciences.

\section{References}

Al-Shabanah OA, Hafez MM, Hassan ZK, et al (2014). Methylation of SFRPs and APC genes in ovarian cancer infected with high risk human papillomavirus. Asian Pac J Cancer Prev, 15, 2719-25.

Anastas JN, Moon RT (2013). WNT signalling pathways as therapeutic targets in cancer. Nat Rev Cancer, 13, 11-26.

Aust DE, Haase M, Dobryden L, et al (2005). Mutations of the BRAF gene in ulcerative colitis-related colorectal carcinoma. Int J Cancer, 115, 673-7.

Azarschab P, Porschen R, Gregor M, Blin N, Holzmann K (2002). Epigenetic control of the E-cadherin gene (CDH1) by $\mathrm{CpG}$ methylation in colectomy samples of patients with ulcerative colitis. Genes Chromosomes Cancer, 35, 121-6.

Barker N, Clevers H (2006). Mining the Wnt pathway for cancer therapeutics. Nat Rev Drug Discov, 5, 997-1014.

Baylin SB, Ohm JE (2006). Epigenetic gene silencing in cancer-a mechanism for early oncogenic pathway addiction? Nat Rev Cancer, 6, 107-16.

Bosch LJ, Mongera S, Terhaar Sive Droste JS, et al (2012). Analytical sensitivity and stability of DNA methylation testing in stool samples for colorectal cancer detection. Cell Oncol (Dordr), 35, 309-15.

Brentnall TA, Crispin DA, Rabinovitch PS, et al (1994). Mutations in the p53 gene: an early marker of neoplastic progression in ulcerative colitis. Gastroenterology, 107, 369-78.
Bronner MP, Culin C, Reed JC, Furth EE (1995). The bcl-2 proto-oncogene and the gastrointestinal epithelial tumor progression model. Am J Pathol, 146, 20-6.

Burmer GC, Rabinovitch PS, Haggitt RC, et al (1992). Neoplastic progression in ulcerative colitis: histology, DNA content, and loss of a p53 allele. Gastroenterology, 103, 1602-10.

Chambers WM, Warren BF, Jewell DP, Mortensen NJ (2005). Cancer surveillance in ulcerative colitis. Br J Surg, 92, 928-36.

Cooke J, Zhang H, Greger L, et al (2012). Mucosal genomewide methylation changes in inflammatory bowel disease. Inflamm Bowel Dis, 18, 2128-37.

deVos T, Tetzner R, Model F, et al (2009). Circulating methylated SEPT9 DNA in plasma is a biomarker for colorectal cancer. Clin Chem, 55, 1337-46.

Dhir M, Montgomery EA, Glockner SC, et al (2008). Epigenetic regulation of WNT signaling pathway genes in inflammatory bowel disease (IBD) associated neoplasia. J Gastrointest Surg, 12, 1745-53.

Fang WJ, Zheng Y, Wu LM, et al (2012). Genome-wide analysis of aberrant DNA methylation for identification of potential biomarkers in colorectal cancer patients. Asian Pac J Cancer Prev, 13, 1917-21.

Fleisher AS, Esteller M, Harpaz N, et al (2000). Microsatellite instability in inflammatory bowel disease-associated neoplastic lesions is associated with hypermethylation and diminished expression of the DNA mismatch repair gene, hMLH1. Cancer Res, 60, 4864-8.

Goel GA, Kandiel A, Achkar JP, Lashner B (2011). Molecular pathways underlying IBD-associated colorectal neoplasia: therapeutic implications. Am J Gastroenterol, 106, 719-30.

Gonzalo V, Lozano JJ, Munoz J, et al (2010). Aberrant gene promoter methylation associated with sporadic multiple colorectal cancer. PLoS One, 5, 8777.

Hanauer SB (2006). Inflammatory bowel disease: epidemiology, pathogenesis, and therapeutic opportunities. Inflamm Bowel Dis, 1, 3-9.

Harpaz N, Ward SC, Mescoli C, Itzkowitz SH, Polydorides AD (2013). Precancerous lesions in inflammatory bowel disease. Best Pract Res Clin Gastroenterol, 27, 257-67.

Hartnett L, Egan LJ (2012). Inflammation, DNA methylation and colitis-associated cancer. Carcinogenesis, 33, 723-31.

Hegde ML, Hazra TK, Mitra S (2008). Early steps in the DNA base excision/single-strand interruption repair pathway in mammalian cells. Cell Res, 18, 27-47.

Herbst A, Wallner M, Rahmig K, et al (2009). Methylation of helicase-like transcription factor in serum of patients with colorectal cancer is an independent predictor of disease recurrence. Eur J Gastroenterol Hepatol, 21, 565-9.

Herrinton LJ, Liu L, Levin TR, et al (2012). Incidence and mortality of colorectal adenocarcinoma in persons with inflammatory bowel disease from 1998 to 2010 . Gastroenterology, 143, 382-9.

Hong L, Ahuja N (2013). DNA methylation biomarkers of stool and blood for early detection of colon cancer. Genet Test Mol Biomarkers, 17, 401-6.

Issa JP, Ahuja N, Toyota M, Bronner MP, Brentnall TA (2001). Accelerated age-related $\mathrm{CpG}$ island methylation in ulcerative colitis. Cancer Res, 61, 3573-7.

Jain SK, Peppercorn MA (1997). Inflammatory bowel disease and colon cancer: a review. Dig Dis, 15, 243-52.

Jawad N, Direkze N, Leedham SJ (2011). Inflammatory bowel disease and colon cancer. Recent Results Cancer Res, $\mathbf{1 8 5}$, 99-115.

Jenke AC, Zilbauer M (2012). Epigenetics in inflammatory bowel disease. Curr Opin Gastroenterol, 28, 577-84.

Kellermayer R (2012). Epigenetics and the developmental 
MGMT-B Gene Promoter Hypermethylation in Patients with Inflammatory Bowel Disease a Novel Finding

origins of inflammatory bowel diseases. Can J Gastroenterol, 26, 909-15.

Konishi K, Shen L, Wang S, et al (2007). Rare CpG island methylator phenotype in ulcerative colitis-associated neoplasias. Gastroenterology, 132, 1254-60.

Lange CP, Campan M, Hinoue T, et al (2012). Genome-scale discovery of DNA-methylation biomarkers for blood-based detection of colorectal cancer. PLoS One, 7, 50266.

Lao VV, Grady WM (2011). Epigenetics and colorectal cancer. Nat Rev Gastroenterol Hepatol, 8, 686-700.

Lin Z, Hegarty JP, Cappel JA, et al (2011). Identification of disease-associated DNA methylation in intestinal tissues from patients with inflammatory bowel disease. Clin Genet, 80, 59-67.

Low D, Mizoguchi A, Mizoguchi E (2013). DNA methylation in inflammatory bowel disease and beyond. World $J$ Gastroenterol, 19, 5238-49.

Matsumura S, Oue N, Ito R, et al (2003). The promoter methylation status of the DNA repair gene O6-methylguanine-DNA methyltransferase in ulcerative colitis. Virchows Arch, 443, 518-23.

Mattar MC, Lough D, Pishvaian MJ, Charabaty A (2011). Current management of inflammatory bowel disease and colorectal cancer. Gastrointest Cancer Res, 4, 53-61.

Mikami T, Yoshida T, Numata Y, et al (2007). Low frequency of promoter methylation of O6-methylguanine DNA methyltransferase and hMLH1 in ulcerative colitisassociated tumors: comparison with sporadic colonic tumors. Am J Clin Pathol, 127, 366-73.

Mokarram P, Naghibalhossaini F, Saberi Firoozi M, et al (2008). Methylenetetrahydrofolate reductase C677T genotype affects promoter methylation of tumor-specific genes in sporadic colorectal cancer through an interaction with folate/ vitamin B12 status. World J Gastroenterol, 14, 3662-71.

Mokarram P, Zamani M, Kavousipour S, et al (2013). Different patterns of DNA methylation of the two distinct O6methylguanine-DNA methyltransferase (O6-MGMT) promoter regions in colorectal cancer. Mol Biol Rep, 40, 3851-7.

Munkholm P (2003). Review article: the incidence and prevalence of colorectal cancer in inflammatory bowel disease. Aliment Pharmacol Ther, 2, 1-5.

Nakagawa H, Koyama K, Murata Y, et al (2000). APCL, a central nervous system-specific homologue of adenomatous polyposis coli tumor suppressor, binds to p53-binding protein 2 and translocates it to the perinucleus. Cancer Res, 60, 101-5.

Noah TK, Lo YH, Price A, et al (2013). SPDEF functions as a colorectal tumor suppressor by inhibiting beta-catenin activity. Gastroenterology, 144, 1012-23.

Okochi O, Hibi K, Sakai M, et al (2003). Methylation-mediated silencing of SOCS-1 gene in hepatocellular carcinoma derived from cirrhosis. Clin Cancer Res, 9, 5295-8.

Olaru AV, Cheng Y, Agarwal R, et al (2012). Unique patterns of $\mathrm{CpG}$ island methylation in inflammatory bowel diseaseassociated colorectal cancers. Inflamm Bowel Dis, 18, 641-8.

Pandurangan AK, Esa NM (2014). Signal transducer and activator of transcription 3-a promising target in colitisassociated cancer. Asian Pac J Cancer Prev, 15, 551-60.

Petronis A, Petroniene R (2000). Epigenetics of inflammatory bowel disease. Gut, 47, 302-6.

Philipp AB, Stieber P, Nagel D, et al (2012). Prognostic role of methylated free circulating DNA in colorectal cancer. Int $J$ Cancer, 131, 2308-19.

Piperi C, Themistocleous MS, Papavassiliou GA, et al (2010). High incidence of MGMT and RARbeta promoter methylation in primary glioblastomas: association with histopathological characteristics, inflammatory mediators and clinical outcome. Mol Med, 16, 1-9.

Psofaki V, Kalogera C, Tzambouras N, et al (2010). Promoter methylation status of hMLH1, MGMT, and CDKN2A/p16 in colorectal adenomas. World J Gastroenterol, 16, 3553-60.

Sangplod P, Kanngurn S, Boonpipattanapong T, Ruangrat P, Sangkhathat S (2014). Expression of BMP6 is associated with its methylation status in colorectal cancer tissue but lacks prognostic significance. Asian Pac J Cancer Prev, 15, 7091-5.

Sato F, Shibata D, Harpaz N, et al (2002). Aberrant methylation of the HPP1 gene in ulcerative colitis-associated colorectal carcinoma. Cancer Res, 62, 6820-2.

Shamsara J, Sharif S, Afsharnezhad S, et al (2009). Association between MGMT promoter hypermethylation and p53 mutation in glioblastoma. Cancer Invest, 27, 825-9.

Summers T, Langan RC, Nissan A, et al (2013). Serum-based DNA methylation biomarkers in colorectal cancer: potential for screening and early detection. J Cancer, 4, 210-6.

Svrcek M, Buhard O, Colas C, et al (2010). Methylation tolerance due to an O6-methylguanine DNA methyltransferase (MGMT) field defect in the colonic mucosa: an initiating step in the development of mismatch repair-deficient colorectal cancers. Gut, 59, 1516-26.

Switzeny OJ, Mullner E, Wagner KH, et al (2012). Vitamin and antioxidant rich diet increases MLH1 promoter DNA methylation in DMT2 subjects. Clin Epigenetics, 4, 19.

Tahara T, Shibata T, Nakamura M, et al (2009). Effect of MDR1 gene promoter methylation in patients with ulcerative colitis. Int J Mol Med, 23, 521-7.

Ullman TA, Itzkowitz SH (2011). Intestinal inflammation and cancer. Gastroenterology, 140, 1807-16.

Wani M, Afroze D, Makhdoomi M, et al (2012). Promoter methylation status of DNA repair gene (hMLH1) in gastric carcinoma patients of the Kashmir valley. Asian Pac J Cancer Prev, 13, 4177-81.

Waris G, Ahsan H (2006). Reactive oxygen species: role in the development of cancer and various chronic conditions. $J$ Carcinog, 5, 14.

Whitehall VL, Walsh MD, Young J, Leggett BA, Jass JR (2001). Methylation of O-6-methylguanine DNA methyltransferase characterizes a subset of colorectal cancer with low-level DNA microsatellite instability. Cancer Res, 61, 827-30.

Widschwendter M, Menon U (2006). Circulating methylated DNA: a new generation of tumor markers. Clin Cancer Res, 12, 7205-8.

Wu JY, Wang J, Lai JC, et al (2008). Association of O6methylguanine-DNA methyltransferase (MGMT) promoter methylation with p53 mutation occurrence in non-small cell lung cancer with different histology, gender, and smoking status. Ann Surg Oncol, 15, 3272-7.

Yin J, Harpaz N, Tong Y, et al (1993). p53 point mutations in dysplastic and cancerous ulcerative colitis lesions. Gastroenterology, 104, 1633-9.

You J, Nguyen AV, Albers CG, Lin F, Holcombe RF (2008). Wnt pathway-related gene expression in inflammatory bowel disease. Dig Dis Sci, 53, 1013-9.

Zhai R, Zhao Y, Su L, et al (2012). Genome-wide DNA methylation profiling of cell-free serum DNA in esophageal adenocarcinoma and Barrett esophagus. Neoplasia, 14, 29-33.

Zhang W, Bauer M, Croner RS, et al (2007). DNA stool test for colorectal cancer: hypermethylation of the secreted frizzledrelated protein-1 gene. Dis Colon Rectum, 50, 1618-26.

Zisman TL, Bronner MP, Rulyak S, et al (2012). Prospective study of the progression of low-grade dysplasia in ulcerative colitis using current cancer surveillance guidelines. Inflamm 
Pooneh Mokarram et al

Bowel Dis, 18, 2240-6.

Zisman TL, Rubin DT (2008). Colorectal cancer and dysplasia in inflammatory bowel disease. World J Gastroenterol, 14, 2662-9. 